\title{
La Jeunesse des Banlieues Françaises De La Violence à la Participation
}

\author{
Véronique Bordes * \\ Alain Vulbeau ${ }^{* *}$
}

\begin{abstract}
$\mathrm{R}$ mo: Este artigo parte dos distúrbios que as periferias parisienses viveram no Outono de 2005. Mais do que uma informação sobre os acontecimentos trata-se de uma reflexão sobre o lugar dos jovens destes bairros periféricos e do reconhecimento político que lhes é atribuído. Após uma pequena síntese dos acontecimentos e do contexto em que surgem, os autores deste artigo constatam o silêncio político destes jovens. Desejando entender o lugar político dos jovens num processo de interacção com os responsáveis territoriais, os autores explicitam os resultados de uma pesquisa-acção levada a cabo com os membros jovens do conselho local de jovens. Essa pesquisa-acção permitiu actualizar temas como o território, a escola, a discriminação, etc. Assim, o artigo convida a ouvir em directo as palavras dos jovens. A pretensão última deste tipo de abordagem investigativa é a conversão da experiência de populaçẽes estigmatizadas em palavras políticas audíveis pelos responsáveis locais e nacionais.
\end{abstract}

Palavras-chave: juventude; precariedade; violência urbana; participação; lugar político da juventude.

\section{Les Émeutes et leur Contexte}

La France a connu pendant plusieurs semaines, de la fin du mois d'octobre a milieu du mois de novembre 2005, des événements que nous appellerons ici les émeutes de l'automne. Ces événements qui ont eu un grand retentissement en France mais aussi à l'étranger ont opposé pendant plusieurs semaines des jeunes habitants des quartiers périphériques des grandes villes de France et les forces de police, essentiellement la nuit avec, pour effet le plus visible, des incendies de véhicules et de bâtiments publics. Puis, au printemps 2006, des grèves étudiantes et lycéennes et de grandes manifestations ont eu lieu pendant presque deux mois, pour refuser une loi qui instaurait un nouveau contrat de travail pour les jeunes de moins de 26 ans. Ces deux mouvements ont été souvent opposés: celui de l'automne serait celui des jeunes marginalisés des quartiers, celui du printemps apparaîtrait comme celui des jeunes des classes moyennes. Des analyses récentes montrent que ces deux mouvements sont distincts par leur localisation et leurs expressions et que des violences ont été commises par certains jeunes des quartiers contre des étudiants et lycéens. Cependant, ces recherches attirent également l'attention sur le climat unitaire qui a marqué les dernières manifestations précédant la suppression de la loi en avril 2006 (Lagrange et Oberti, 2006).

Dans ce contexte, le point de départ de cet article se situera plus en relation avec les événements de l'automne 2005. Le premier constat est que ces émeutes ne sont pas les premières et sans doute pas les dernières. Depuis les évènements survenus dans la banlieue de Lyon en 1981, des manifestations de ce genre ont eu lieu régulièrement, dont l'origine a pratiquement toujours été la mort de jeunes hommes causée par la police (Bachmann et Guennec, 1996, 1997). Les émeutes de l'automne ont eu également pour point de départ, le décès de deux adolescents de 15 et 17 ans à

\footnotetext{
* Docteure em sciences de l'éducation, chercheure associée au CREF, Centre de Recherche Éducation et Formation, équipe Crise, Écoles, Terrains sensibles, Université Paris 10 Nanterre. Contact: bordesveronique@wanadoo.fr

** Professeur de sciences de l'éducation, chercheur au CREF, Centre de Recherche Éducation et Formation, équipe Crise, Écoles, Terrains sensibles, Université Paris 10 Nanterre. Contact: alain.vulbeau@wanadoo.fr
} 
Clichy-sous-Bois en Seine-Saint-Denis (dans la banlieue nord de Paris), qui, fuyant un contrôle de police, s'étaient réfugiés sur le terrain d'un transformateur d'électricité et sont morts brûlés alors qu'un troisième jeune était grièvement blessé. Un processus émeutier s'installe pendant trois semaines qui va connaître plusieurs phases ${ }^{1}$. La première phase est localisée dans la commune de Clichy-sous-Bois, la seconde phase s'étend à toute la région parisienne, la troisième s'étend à toute la France avec environ 300 communes concernées.

Au cours des émeutes de l'automne il y aura un mort, en plus des deux jeunes déjà cités, et environ 220 blessés parmi les policiers et les pompiers. On ne dispose pas de chiffres concernant les jeunes émeutiers. Le bilan des pertes matérielles est particulièrement lourd puisque l'on compte 10000 véhicules incendiés et des centaines de bâtiments publics (écoles, crèches, gymnases ainsi que quelques établissements religieux) partiellement endommagés et dans quelques cas, totalement détruits par le feu. A la fin du mois de Novembre 2005, plus de 5000 personnes sont interpellées par la police et 800 sont emprisonnées.

Le ministère de l'intérieur proposera deux séries d'explication à ces émeutes. Dans un premier temps, il sera question de bandes organisées en relation avec la mouvance islamique radicale mais cette thèse sera contredite publiquement par les Renseignements Généraux (police politique française); une seconde explication tentera de rabattre ces émeutes sur la problématique de la délinquance et plus particulièrement sur le thème de la "violence gratuite". Ainsi cette violence serait une conduite sans explication rationnelle, causée par la mauvaise éducation familiale des jeunes et, en définitive, des tendances criminelles innées, renforcées par les dimensions pathogènes de l'urbanisme des quartiers d'habitat social.

La plupart des ouvrages de recherche, notamment ceux publiés dans le contexte des émeutes insistent sur différents facteurs explicatifs relevant des domaines sociaux, urbains et politiques.

Au plan social, les jeunes de ces quartiers sont quatre fois plus exposés au chômage que la moyenne de la population française et, deux fois plus que la moyenne des jeunes. Le chômage est intégré à un mode de vie précarisé où se succèdent des stages non rémunérés, des "petits boulots" plus ou moins officiels et, dans certains cas, des trafics relevant d'une délinquance plus ou moins aggravée. Parfois, cette précarité prend place dans un contexte local de récession industrielle comme l'illustrent les émeutes de Montbéliard en 2000 (Beaud et Pialoux, 2003). Le racisme et diverses formes de discrimination sont la toile de fond de cette précarité, alors que ces phénomènes sont peu reconnus et peu combattus par les pouvoirs publics. Ceux-ci en sont parfois les auteurs comme le montrent les conflits incessants entre jeunes des quartiers et policiers. Le moment le plus emblématique de ces relations est le contrôle d'identité qui, sous couvert d'une simple vérification, parfois reproduite à plusieurs reprises dans une même journée, donne lieu à des mises en cause de la réalité de la nationalité française des jeunes.

Les raisons urbaines participent d'un débat, récurrent en France depuis plus d'une trentaine d'années, sur la ségrégation, en lien avec l'urbanisme des grands ensembles d'habitat social, construits dans les années 1960 et 1970. Les composantes de ce débat mentionnent une série de dysfonctionnements urbanistiques: enclavement des cités dans des zones périphériques, faiblement dotées en transports collectifs; mauvaise qualité initiale et mauvais entretien des immeubles par les bailleurs; population très homogène due au regroupement forcé des communautés étrangères ou d'origine étrangère, etc... Pour une part, ce débat alimente les politiques de développement social et urbain (ou politique de la Ville). Le traitement de ces processus socio-urbains par cette politique fait l'objet d'interrogations régulières quant à ses domaines d'intervention, ses moyens financiers, son efficacité, etc. (Vieillard-Baron, 2001).

Les raisons politiques renvoient aux marges de manoeuvre de trois catégories d'acteurs: l'État, les collectivités locales, en particulier les municipalités, et les habitants des quartiers. Le maire est de plus en plus l'acteur central et la politique de rénovation urbaine, est mise en place à partir de la commune. L'équipe opérationnelle de maîtrise d'oeuvre urbaine qui, autrefois était indépendante, est désormais placée sous la responsabilité du

\footnotetext{
1 Nous empruntons largement le récit des événements à "Les émeutes de novembre 2005" texte de Mucchielli L. et Aït-Omar A. qui introduit le livre dans: Mucchielli L., Le Goaziou V.(dir), 2006, Quand les banlieues brûlent: retour sur les émeutes de novembre 2005, Paris, La Découverte.
} 
maire. L'État est de plus en plus, selon la formule de Jacques Donzelot, un "État-animateur" dont la mission est moins de déterminer l'action publique que de la réguler. Quant aux habitants, il faut constater qu'ils sont au coeur d'un double discours de la part de l'État et des collectivités locales. D'un côté, ils sont déclarés au centre du dispositif: tout est fait pour les habitants et tout devrait être fait avec leur participation. D’un autre côté, le pouvoir qui devrait découler de ces principes n'est jamais assuré par des mesures institutionnelles: 1" "empowerment" des habitants ne prend pas forme dans ces quartiers qui ne sont pas perçus comme une unité politique mais seulement comme une unité d'action administrative.

\section{Silence ou expression des jeunes?}

Dans le contexte localisé des émeutes et celui plus globalisé de l'environnement social, urbain et politique, s'intéresser à la place des jeunes impose la prise en compte de facteurs multiples, cependant, nous allons insister ici sur deux points particuliers: l'expression des jeunes des quartiers et leur constitution en interlocuteurs politiques. Si l'on prend en compte la dimension des générations, on voit que l'empowerment juvénile existe avec le dispositif des conseils de jeunes mis en place par certaines municipalités. Le silence des jeunes pendant les émeutes a souvent été souligné par les observateurs ou les médias qui ont noté l'absence de revendications explicites. En fait, il y a bien eu une revendication fondée sur la mise en cause personnelle du ministre de l'intérieur qui avait employé des qualificatifs stigmatisants comme "racaille", "gangrène" à l'égard des jeunes, peu de temps avant les émeutes ${ }^{2}$.

Cette demande de respect est bien une revendication que l'on peut retrouver dans le document qui a circulé par mail pendant les émeutes. Dans le texte, "Nous jeunes de Stains", daté du 9 Novembre 2005 et cité ci-dessous, s'exprime le point de vue des membres du conseil local de jeunes de Stains, petite ville de la banlieue nord de Paris, proche de Clichy-sous-Bois, point de départ des émeutes.

\section{NOUS JEUNES DE STAINS...}

...membres du Conseil Consultatif de la Jeunesse (CCJ), nous demandons que la vérité soit révélée et la justice rendue car Bouna et Ziad sont décédés dans des conditions qui doivent être élucidées.

Ce drame est la goutte d'eau qui a fait débordé le vase du malaise profond que nous vivons dans notre quotidien. Nous ne supportons plus les conditions de chômage, de crise du logement, de dégradation de notre environnement dans lesquelles nous vivons.

Nous aspirons à une dignité et au respect des libertés et des droits fondamentaux. De même que le droit à l'éducation, à la formation et à l'emploi pour tous.

Attentes d'autant plus légitimes qu'elles concernent l'ensemble de la population française comme le prouve l'étendue des évènements à toute la France.

Dans le même temps, nous ne pouvons accepter la destruction des équipements publics qui sont à notre service ainsi que le climat de tension et de peur qui règne et qui risque de faire le lit de l'intolérance.

L'image véhiculée par les médias et les propose méprisants du ministre Sarkozy nous sont intolérables et dégradants.

Nous sommes des citoyens à part entière et en avons assez des étiquettes: racaille, voyou, délinquant... A ce titre, nous vivons tous les jours des moments de solidarité et d'entraide dans nos lycées, associations, familles et quartiers. Et c'est cela que nous voulons aujourd'hui valoriser et partager.

Nous lançons un appel à nous rencontrer afin de continuer à construire et avancer ensemble pour notre bien-être à toutes et à tous.

\footnotetext{
${ }^{2}$ Le centre de ressources de la politique de la ville de l'Essonne a rassemblé un dossier de presse et une bibliographie sur ces émeutes: http:/ /ressourcespolville.maisondebanlieue.asso.fr
} 
Ce message montre que le silence des jeunes est relatif et conduit à une question: le silence est-il le fait d'une absence de paroles ou l'effet d'une absence d'écoute? Répondre à cette question suppose de se demander que disent les jeunes concernés par la précarité et la stigmatisation. Les auteurs de cet article souhaitent contribuer à la connaissance et à l'écoute de ce public en relatant des opinions présentées dans le cadre d'une recherche-action à laquelle certains jeunes signataires du texte "Nous Jeunes de Stains" ont participé.

De septembre 2002 à mai 2003, le centre de ressources Profession banlieue ${ }^{3}$ en collaboration avec les éditions de l'Atelier, a décidé de réunir des jeunes de Seine-Saint-Denis pour échanger sur leur usage de la ville. À ce groupe se sont ajoutés des représentants de collectivités locales et des institutions concernées par la question juvénile au plan local et national. Deux membres du centre de ressources, l'éditeur et deux chercheurs ont constitué l'équipe opérationnelle de ce projet. Ce travail de recherche-action avait pour but de faire un ouvrage "sur" les jeunes mais "avec" eux (Bordes et Vulbeau, 2004).

Les jeunes, garçons et filles, tous volontaires, habitent le département de la Seine-Saint-Denis, sont pour la majorité français issus de l'immigration maghrébine et africaine et ont pratiquement tous suivi des études supérieures. Ils interviennent dans un dispositif de participation ouvert aux jeunes (conseil local, départemental ou national de la jeunesse) et sont, pour certains, élus de conseils municipaux de communes communistes ou d'union de la gauche. Des réunions thématiques ont permis des discussions sur des sujets comme le territoire, l'éducation, la politique, le travail ou des processus comme la discrimination ou la participation. Une réunion spéciale pour les jeunes femmes a été rajoutée, avec l'accord du groupe, afin de rendre plus explicites leurs points de vue et la question de la place des femmes.

Les débats ont été enregistrés puis décryptés, ce qui a permis aux chercheurs de faire un travail classique d'analyse de discours fondé sur des éléments objectivés. Des versions successives ont été présentées au groupe qui a participé par la critique aux diverses phases de la réalisation de l'ouvrage. L'ensemble de ces opérations a permis de déboucher sur le manuscrit final après des débats qui ont parfois révélé des contradictions. Les chercheurs, se positionnant comme des "écrivains publics", ont tenté de maintenir un équilibre entre les exigences d'une production de recherche et la volonté d'être au plus près de la parole, mais aussi de l'analyse que faisaient les jeunes de leur propre situation. Finalement, cette recherche devait permettre de porter un regard plus ajusté et plus lucide sur une jeunesse hétérogène et désireuse de s'occuper du bien commun.

Dans la suite de cet article, nous souhaitons présenter au lecteur une synthèse de ce travail: les dispositifs de participation sont des lieux conflictuels où le personnel politique n'apparaît pas toujours sous son meilleur profil. Seule la prise en compte directe de l'avis des jeunes pouvait nous faire comprendre ce que valent les propositions de participation et comment ces dernières traitent leurs problèmes au plus près de la façon dont ils se les représentent. Des jeunes qui s'avèrent des ressources pour leur territoire, ont pris la parole en s'inscrivant dans une offre politique locale. Ils se sont impliqués dans des actions de lutte contre les discriminations s'inscrivant dans des formes d'engagement qui n'étaient pas toujours attendus par les adultes, découvrant du même coup des rapports de force là où ils pensaient simplement exercer une citoyenneté ordinaire.

Cette parole, que certains ont présentée comme déficiente ou inaudible durant les événements de l'automne 2005, témoigne d'une alternative citoyenne en s'adressant aux personnes qui ne veulent pas se laisser assourdir par le tintamarre du discours sécuritaire. Pour saisir toute l'importance de cette parole des acteurs, il a été décidé de la faire apparaître en italique pour la distinguer des propos des auteurs de l'article ou d'autres formes de citation.

\section{Le Soupçon et la Menace}

Lors des échanges au sein du groupe, un certain nombre de thèmes ont été abordés. La première interrogation a été posée autour du mot

\footnotetext{
${ }^{3}$ Le centre de ressources Profession Banlieue, créé en 1993, a bout but de qualifier les professionnels du développement social urbain (chefs de projet, médiateurs, agents de développement, femmes-relais, etc) du département de Seine-Saint-Denis
} 
«jeunesse». Ce terme est marqué par un certain nombre de représentations qui participe à des positionnements de soupçons face à une menace potentielle.

Le mot «jeune» ne laisse jamais indifférent et reste à l'origine d'un certain nombre de représentations. On parle toujours du même type de jeunes. Depuis tout à l'heure, on parle du jeune du quartier, de la cité, plutôt maghrébin, et plutôt issu d'un milieu défavorisé. Si on se penche sur les propos du groupe, de nombreuses questions se posent alors autour de la notion de jeunesse. La question récurrente étant de savoir qui est jeune et qui ne l'est pas.

La jeunesse ne peut plus être considérée comme un simple passage d'un état vers un autre, mais bien comme un processus de construction des individus. En se socialisant, la jeunesse intègre, par le biais de tâtonnements et d'expérimentations, une place construite au travers d'un trajet social fait d'apprentissages, d'échanges et de créations.

De leur côté, les institutions s'emploient à donner une définition de la jeunesse en enfermant les âges dans des dispositifs cadrés et calibrés, déterminant selon une logique qui leur est propre, qui est jeune et qui ne l'est plus, allant même jusqu'à catégoriser les jeunes entre eux.

Pourtant, la catégorie «jeune» n'est pas un effet de désignation et peut être revendiquée comme une qualité. Moi aujourd'hui, j'ai 29 ans, je me considère toujours comme un jeune. Je pense que je suis jeune! Je suis jeune!. Mais la jeunesse reste fragile, considérée comme une tranche d'âge et non comme une catégorie sociale, ce qui fait oublier qu'elle peut être sans ressources, démarrant dans la vie, pour certain, avec un handicap social dû aux origines ethniques, géographiques, mais aussi socioprofessionnelles.

Au sein de la jeunesse, les filles tiennent une place particulière. Leur vision de la ville, leur usage des espaces publics et leur obligation à gérer «l'exposition» féminine induisent la nécessité de développer des capacités d'adaptation au milieu où elles évoluent, différentes de celles des garçons. Quand je vais sur Paris, j'ai envie d'être tranquille. Et à partir du moment où je n'ai pas envie qu'on me prenne la tête dans le RER, ça va être jeans et baskets. Ces capacités d'adaptation sont aussi nécessaires dans leur relation avec les garçons. Il faut à la fois pouvoir tenir sa place de fille, sans être cataloguée de fille qui craint, mais en conservant une place au sein du groupe de pairs.

Cette construction d'une image joue un rôle dans l'apparition de représentations autour d'un «jeune de banlieue» souvent présenté comme disqualifié, dangereux et en danger. Le fait de vivre en périphérie d'une grande ville, dans un grand ensemble, d'être issu de l'immigration et donc d'une autre culture, participe à la construction de stigmates disqualifiant difficiles à surmonter. Si un jeune se dit: moi, je vais me casser la tête à montrer que je suis quelqu'un de bien, un autre lui réplique que s'il est stigmatisé c'est parce que tu as la tête. De leur côté, certaines institutions se servent d'images construites pour leur propre publicité, laissant paraître un intérêt pour une certaine jeunesse, mais mettant finalement en scène des caricatures stigmatisantes. Les jeunes de banlieue sont alors perçus comme des déviants potentiels nécessitant la mise en place d'une politique de répression. C'est ainsi que les politiques développées en direction de la jeunesse deviennent des «cadres» de prévention dans lesquels ont ne répond plus aux besoins de la jeunesse, mais où on tente de préserver une certaine «paix sociale». Et lorsque la jeunesse revendique une véritable place d'acteur social, elle passe de l'image d'une «jeunesse dangereuse» à celle de «jeunesse en danger». La notion de représentation est alors utilisée, manipulée dans un double usage de discrimination et de valorisation, au gré des intérêts de chacun.

Les événements de l'automne 2005 puis les manifestations anti-CPE du printemps 2006 sont un bon exemple de cette construction d'une image d'une jeunesse qui peut passer indifféremment de la catégorie "dangereuse" à "en danger".

Lorsque les banlieues se sont embrasées, portant sa jeunesse sur le devant de la scène publique de la même façon que dans les années 1980, puis 1990, les responsables politiques ont voulu montrer que la banlieue était habitée de jeunes «sauvageons», échappant à toute éducation, ne connaissant pas les lois et oubliant leur devoir de citoyen. La jeunesse «dangereuse» est apparue comme le nouveau mal des banlieues. Un discours politique a développé alors la nécessité de rétablir l'ordre dans les périphéries en se fondant sur des mesures de répression. Relayés par certains médias, des responsables politiques ont développé une stratégie consistant à faire une apparition dans 
un quartier, prendre à partie la population pour pouvoir appuyer ensuite des actions répressives contre la jeunesse. Le fond même des actions de la jeunesse des banlieues a été manipulé pour que la population ressente un fort sentiment d'insécurité envers sa propre jeunesse. Loin de constater l'échec de la politique sociale en banlieue, les responsables ont promis d'agir pour supprimer une violence née de la discrimination.

Lorsque les jeunes sont descendus dans la rue pour protester contre une loi qui entraînait une progression inévitable de la précarité, déjà bien installée chez les jeunes, ceux-ci ont été présentés comme «en danger». D’abord parce que, soi-disant manipulée par des forces cachées, la jeunesse ne pouvait être perçue comme capable de développer un quelconque esprit critique. Ensuite, parce que trop jeune, trop idéaliste pour comprendre l'importance de son avenir, la jeunesse était pour quelques-uns, par tradition, inconsciente des enjeux. Enfin, parce que sa place, pour certains, devait être à l'école et non dans la rue qui n'est pas considérée comme un espace éducatif où construire son avenir.

Pourtant, cette jeunesse que l'on veut nous présenter comme "fragile et en danger" est consciente de la nécessité de participer pour obtenir une place. En se mettant en scène, que ce soit dans les banlieues ou dans les rues des centres villes, la jeunesse montre que, au-delà du soupçon de la menace, elle est en capacité de participer de façon citoyenne à la vie de la cité, dénonçant les discriminations dont elle est victime, par l'expression de signes forts d'une volonté de changement. Ces manifestations de la jeunesse, si elles apparaissent comme des actes désespérés, sont aussi la preuve de l'existence d'une envie de prendre place dans le jeu social. Pourtant, le plus souvent, l'ombre de la discrimination plane sur l'ensemble de la jeunesse, et plus particulièrement sur celle des banlieues.

\section{Territoire et Discrimination}

Le territoire des jeunes est un espace de vie complexe où on trouve des lieux de séjour et des lignes de mobilités. Il relève d'une géographie affective et identitaire qui sait s'ouvrir à l'exploration d'autres mondes. Des caractéristiques opposables deux à deux permettent de décrire cet espace: la proximité et la distance, la sédentarité et la mobilité, l'interaction avec les autres générations et le confinement dans l'entre-soi des pairs.
L'espace des jeunes vivant dans des grands ensembles en périphérie de Paris est d'emblé limité par des barrières financières. Dehors, on a l'impression qu'il y a une zone citoyenne, de centreville avec de belles choses, dedans, c'est la zone où l'on stocke un certain nombre de personnes, dont la seule activité est de tuer le temps.

L'espace n'est pas seul en jeu dans ces problèmes d'enfermement. Les représentations construites autour de cet espace tiennent un rôle. Si les jeunes sont enfermés dans leurs quartiers, ils subissent aussi les effets des appellations attribuées à leur habitat. La notion de «quartiers sensibles», si elle devrait permettre de repérer une attention des pouvoirs publics, devient vite stigmatisante, générant une mise à distance symbolique de certaines populations. On mesure, a contrario, cette connotation négative des terminologies institutionnelles quand on les retournes en se demandant comme un travailleur social: Où sont les quartiers insensibles? les banlieues froides? où sont les zones de bon-droit?

Dans l'espace des cités se déploie une forme de vie spécifique où les cultures des populations servent de normes locales. L'usage des espaces publics est réapproprié dans une perspective de régulation sociale pas toujours perçue comme telle par les personnes extérieures à la cité. Par exemple, dans notre quartier, on voit souvent des enfants maliens qui restent très tard dehors. Ça à tendance à choquer des personnes. Moi personnellement, ça ne me choque pas parce que dans la culture malienne, les enfants sont éduqués par les voisins... Tout le monde éduque les enfants qui sont dehors. Moi quand je suis au pays, ce ne sont pas mes parents qui vont m'éduquer, c'est les voisins, le cousin ou n'importe qui rencontré dans la rue, s'il me voit faire une bêtise.

Cette dimension de surveillance du voisinage est souvent mal comprise, le reproche récurrent que l'on fait aux familles étant la démission éducative. Pourtant, la rue peut être considérée comme un espace de vie représentatif de l'identité du territoire. C'est un lieu où l'on communique sur sa culture, c'est un espace de sociabilité au même titre qu'un court de tennis ou un terrain de golf. Le détournement de l'usage de l'espace public pose le problème de la visibilité de certains groupes sociaux. Ainsi, le stationnement des jeunes dans les halls d'immeubles est devenu un enjeu dans le discours 
sécuritaire, alors qu'il peut s'expliquer comme un espace de dégagement pour des familles nombreuses. Moij'ai été alerté par un sociologue malien qui nous a expliqué que ces jeunes, s'ils sont au pied des escaliers, c'est que, lorsqu'ils rentrent de l'école, ils sont huit ou neuf dans l'appartement, que les parents sont là ou pas là, qu'ils regardent la télé et qu'un, deux ou trois de la famille, spontanément ou normalement, préfèrent rester dehors que rentrer. Nous ne sommes plus dans une situation d'abandon, mais bien dans une alternative au mal être domestique.

Le stationnement dans les halls révèle aussi une impossibilité d'aller en ville. Le fait de rester sur place est presque un marqueur de l'agressivité de la ville à leur égard. La cage d'escalier devient un lieu de socialisation que la ville leur refuse, un espace intermédiaire à l'accès libre qui subsiste. Ce qui nous manque, je le vois sur Stains, ce sont des lieux de vie, en dehors des quartiers, des espèces de placettes sympas, des petits cafés et tout ça. Certains jeunes ont alors du mal à sortir de ces territoires.

Une des portes de sortie possible reste l'école. Moi je sais que le seul moyen de sortir de mon quartier, c'était l'école. Quand je suis parti au lycée, c'est là que j'ai commencé à partir de mon quartier, à aller voir un petit peu ailleurs, à connaître d'autres gens et ensuite à découvrir d'autres endroits. Mais, le processus de réussite scolaire entraîne une mobilité qui rend invisibles les jeunes qui réussissent et qui progressivement, font leur vie ailleurs.

Pourtant, la mobilité scolaire n'arrange pas tout et est à l'origine d'un phénomène de «couloir obligé» qui maintient les jeunes dans l'entre-soi. Moi par exemple, j’ai été à l'école à Saint-Denis, j'ai rencontré d'autres gens, d'accord, mais mon espace ne s'est pas agrandi pour autant. Peut-être qu'en grandissant, on change d'espace, mais on n'agrandit pas notre espace de vie. La mobilité dans une zone restreinte, voire contrainte reste une réalité pour les jeunes. Il y a un circuit qui fait que quand tu es aux 40000 (cité de la banlieue Nord parisienne) et que tu es à Joliot-Curie en primaire, tu iras au collège Jean-Vilar, tu iras peut-être à Jacques Brel (collège) si tu as de la chance. Et puis après c'est Paris VIII ou Paris XIII (universités). Cet effet d'assignation à un certain parcours scolaire débouche sur un sentiment de dévalorisation.
En dehors de l'école, pour sortir des espaces trop confinés que sont les quartiers, il faut avoir des centres d'intérêts qui auront à voir avec l'autonomisation de la jeunesse. Pourtant, quand cette autonomie n'existe pas, il faut la provoquer. C'est peut-être une des fonctions du travail social ou de la politique locale. Un conseil local de jeune peut être un exemple d'espace suffisamment ouvert et attractif pour que les jeunes soient au rendez-vous. A Stains, on a fait le choix de réunir les séances au sein de la mairie. On aurait pu se dire «ça ne va pas marcher, ils ne vont pas sortir de leur quartier». Eh bien la preuve, c'est que ça marche! ils viennent de tous les quartiers. Le territoire n'est plus pensé comme un simple espace d'expression des sociabilités, mais bien comme un espace politique et d'apprentissage politique.

Pourtant, les effets de «couloir» sont tenaces et malgré la volonté de sortir, la mobilité ne semble pas être un processus suffisant. Quand j'avais 18-19 ans, je suis parti en vacances dans un club. On était trois amis et on s'est dit: «chouette, on va partir dans un club de vacances et on ne va rencontrer personne». A quatre heure du matin, on se fait accueillir par les jeunes de Saint-denis et de La Courneuve qui nous font plein de signes comme ça «bonjour, bienvenue!». Ça nous a choqués.

Les jeunes ont tendances à partager systématiquement les «bons plans», la plupart étant proposés par les municipalités, ce qui provoque le déplacement des jeunes d'un lieu vers un autre, sans pour autant brasser la population.

De leur côté, les pouvoirs publics ont œuvré pour la mobilité des jeunes en développant le principe de la carte Imagin'R. Elle permet aux jeunes inscrit dans un établissement scolaire, de se déplacer vers les établissements scolaires ou dans le cadre des loisirs. Pour les autres, sortis du système scolaire, la mobilité devient plus difficile.

La mobilité est aussi en relation avec le genre. Si les garçons développent des mobilités de proximité, les filles s'éloignent plus facilement de leur quartier et de la surveillance informelle des «grands frères».

Le rapport du territoire est donc ambivalent passant de la proximité à la distance, de la sédentarité à la mobilité, les jeunes investissant des espaces par défaut. Cet enfermement spatial a des effets pervers sur la jeunesse qui est renvoyée systématiquement dans un certain entre-soi discriminant. Lors des événements de l'automne 2005, 
les jeunes ont montré combien l'enfermement dans les quartiers était tenace, maintenant leurs actions sur les lieux même de leur habitat. Cette dimension est d'autant plus forte quand on observe les manifestations des étudiants contre le CPE qui n'ont pas hésité à investir les centres-villes. Cette différence d'usage des espaces publics est révélatrice de l'enfermement dans lequel les jeunes peuvent se retrouver au sein même du lieu où ils vivent.

Habiter en banlieue, dans un grand ensemble HLM n'implique pas seulement de subir un environnement souvent dégradé, mais aussi d'être maintenu, d'une certaine manière, à la marge de la société. La discrimination s'exprime dans différents domaines: l'habitat, l'origine ethnique, l'âge ou la qualité sociale. Parmi les participants du groupe, elle est ressentie comme prégnante depuis leur plus tendre enfance, marquant les parcours de formation, mais aussi lors de l'entrée dans le monde du travail.

La famille puis l'institution scolaire sont des lieux de socialisation. Lorsqu'une personne habite un quartier, le parcours scolaire la maintient dans un espace de l'entre-soi. De l'école maternelle à la fin du collège, le système de la carte scolaire inscrit l'enfant dans les écoles de son quartier. Certains groupes scolaires sont situés géographiquement au centre des bâtiments de la cité. L'enfant est donc assigné à un espace dans lequel il ne rencontre que des semblables. L'entrée au lycée est souvent la première occasion pour le jeune de quitter son quartier. Jusqu'au Bac, je n'étais quasiment entouré que de blacks et de beurs. C'est dur à dire, ça. Et puis, j'ai fait un BTS, et là, il y avait des gens qui venaient de Meaux, de Paris et sur une classe de trente-deux élèves, ont été deux maghrébins. Je n'ai pas compris, merde c'est quoi ce truc!

Ce changement de situation entraîne, pour le jeune, un début de prise de conscience sur les différences que créaient les discriminations. Cette réflexion le place devant ses manques et ce double effort qu'il doit fournir pour avancer. Alors je me suis dit "on m'a empêché de voir ce qu'était réellement la société française». C'est après le Bac que j’ai commencé à aller à Paris, etc. Le fait de suivre une formation universitaire ne fait qu'affirmer cette prise de conscience. À un moment donné, tu côtoies malgré tout les mêmes types de personne. Après, c'est le regard que tu portes sur ce que tu es et ce qu'on te renvoie. Reconnais que quand tu dis: je suis à la Sorbonne, et tu dis: je suis à Paris Nord ou à Paris
XIII (Universités de la banlieue Nord de Paris), dans le conscient ou l'inconscient, pour le collectif, ça ne fait pas le même effet. On en revient à cette discrimination qui semble inscrite dans le parcours scolaire de certains jeunes issus «des quartiers». Qu'en est-il alors de l'égalité des chances?

Pourtant, les familles ont un grand respect de l'école et les jeunes sont conscients de l'importance de la formation, même si un même diplôme n'est pas valorisé de la même façon pour un jeune issu d'une cité et un jeune vivant en centre ville. Finalement, le recrutement que l'entreprise (la $\mathrm{SNCF})$ fait en banlieue est toujours à une échelle relativement basse du point de vue hiérarchique, mais du point de vue des cadres, le recrutement ne se fait pas dans ces espaces. Les jeunes se retrouvent face à des offres d'emploi régies par les origines ethniques et non plus par les capacités à intégrer un poste. Les diplômes deviennent contre-productifs. Pour les non diplômés, la «discrimination positive» permet d'obtenir un statut professionnel. Moi j'ai connu la discrimination positive. On m'a embauché parce que j'étais issue de l'immigration maghrébine. Je savais que j'allais servir d'alibi à une politique. Il n'empêche que professionnellement, j’ai tenu à dire: je suis professionnelle, je suis là parce que je suis professionnelle.

La discrimination montre donc deux visages pour ces jeunes vivant dans des cités: le côté «positif» que certains jeunes sans diplôme savent utiliser et le côté «négatif» qui peut soit rejeter simplement un diplômé à cause de son origine, soit permettre l'embauche de personne ressemblant aux usagers de l'entreprise. Les jeunes doivent alors développer des savoir-faire qui leur permettent d'utiliser à leur avantage, les fonctionnements de la discrimination à l'embauche.

\section{Une offre de participation sous influence}

Depuis les émeutes des années 1980-1990 et l'apparition de la qualification de "zones sensibles" pour désigner ces quartiers, un certain nombre de mesures ont été développées pour impliquer les habitants dans la vie locale. Un jeune du groupe explique: c'est vrai que ces dernières années, on a assisté à pas mal de consultations auprès de la population pour diverses raisons, notamment parce que, dans le cadre des conseils de 
quartier, la loi maintenant l'oblige. C'est vrai que c'est un peu à la mode d'aller demander l'avis de... pour construire avec! Souvent, il y a des réponses très formatées. Les jeunes aussi sont concernés par cette consultation. Pourtant, selon un discours récurrent, ils sont difficilement mobilisables. Cette difficulté semble pourtant cacher une autre réalité: la peur de la jeunesse. Les représentations qui entourent les jeunes marquent au quotidien la méfiance des institutions et du politique. Un adjoint au maire participant au groupe nous fait remarquer que laisser les jeunes intégrer l'espace politique c'est aussi intégrer du danger politique, parce que, un jeune, ce n'est pas forcément quelqu'un qui va être de la même couleur politique.

De son côté, la jeunesse reproche aux adultes de les consulter alors que les décisions sont déjà prises. On a été consulté pour un réaménagement d'un centre commercial. On a été consulté pour un espace en bas du bâtiment. Finalement, quand on regarde l'espace qui est construit, ça n'a rien à voir avec ce que les jeunes voulaient.

Certains élus, dans certaines municipalités, ont décidé de réfléchir à la place qu'ils pouvaient donner à la jeunesse. Cette proposition de rencontre entre les jeunes et le politique se retrouve au sein de différentes formes d'offres citoyennes, parmi lesquelles, les conseils de jeunes.

L'accès à la citoyenneté est un thème récurrent dont se sont saisis les responsables politiques comme d'une solution aux maux d'une génération. La forte présence, dans ce groupe, de jeunes inscrits ou ayant été inscrits dans des conseils de jeunes nous permet d'établir un diagnostic précis sur cette offre de participation. Trois espaces leur sont accessibles: le conseil local qui fonctionne au niveau de la commune, le conseil départemental et le conseil national.

Le conseil national des jeunes leur permet de rencontrer des représentants du gouvernement et d'approcher le jeu politique. On avait pour interlocuteurs des ministres ou des directeurs de cabinet, mais cela ne nous empêchait pas de dire les choses directement et parfois de manière assez crue, pour autant, cela ne voulait pas dire qu'après, il y avait action!

Le conseil départemental est une autre forme d'offre de participation. En Seine-Saint-Denis, il a été créé officiellement le 30 janvier 1998. Ces conseils départementaux de la jeunesse sont portés par une volonté politique de voir les jeunes se réunir, réfléchir et essayer de faire un pas vers les institutions et les entités politiques.

Le conseil local des jeunes est présenté par les politiques comme une action complémentaire dans l'instauration d'un dialogue déjà entamé au sein du conseil national de la jeunesse et des conseils départementaux, le dialogue et les initiatives des jeunes apparaissant comme plus faciles au niveau communal.

En écoutant les témoignages des jeunes, quel que soit l'espace dans lequel ils s'investissent, on constate qu'ils développent des compétences qui n'étaient pas envisagées au départ par les institutions. Ils sont capables de se saisir d'une problématique, de l'analyser puis de faire des propositions construites qui peuvent, par leur précision, dérouter les adultes. Ils montrent leur capacité à adapter leur participation, tout en se formant et en utilisant leur compétence urbaine. Ils deviennent des «experts» des problèmes qu'ils rencontrent, du diagnostic à la résolution. Moi je parle de l'expérience du conseil local de jeunes. Depuis deux ans, on se réunit. Pour moi, on a eu une action militante. On a essayé de parler. On l'a fait. On a été clair sur ce qu'on avait envie de dire, mais ensuite, est-ce que le politique a vraiment envie que nous, on se politise?

On constate que ces organismes consultatifs, mis en place pour permettre la participation des jeunes, sont en permanence dans l'expérimentation. Les adultes présentent une réelle envie d'associer les jeunes mais restent toujours angoissés par la peur de l'inconnu, du débordement et de la prise de pouvoir par les jeunes. Ces craintes s'expliquent, en partie, par la méconnaissance du public jeune. Paradoxalement, si ces tâtonnements, ces tentatives, ces indécisions sont gérées difficilement par les élus, cela n'atteint ni les motivations des jeunes, ni leur désir de participation citoyenne. L’exemple du conseil national de la jeunesse où la parole des jeunes semblait "filtrée" par les adultes permet de saisir cette détermination à participer. $I l$ y avait des groupes dissidents qui se disaient: «Cet après-midi, à un moment donné, comment peut-on faire pour prendre le micro?» et à un moment donné je disais «Cet après-midi, je prends le micro et je déballe tout» et toi tu disais «à mon avis ça ne va pas être possible, parce que vu comment les choses sont organisées, on ne te donnera jamais le micro!». 
Cette volonté de s’investir et de participer s'est révélée avec les manifestations du printemps contre le CPE. L'organisation même du mouvement s'est construite petit à petit, passant du tâtonnement à l'affirmation, sans que pour autant les jeunes ne perdent de vue la nécessité de ne prendre place de façon maîtrisée.

De même, les événements des banlieues de novembre ont redonné une certaine visibilité à une jeunesse rendue muette par le jeu de la précarité et de la relégation. Et même si les médias ont essentiellement relayé les images d'incendies de véhicules, certains jeunes étaient présents pour dire la nécessité d'avoir des espaces de parole et de participation citoyennes, comme en témoigne le document des jeunes de Stains, cité au début de cet article. Ces espaces se sont fait rares ces derniers temps, la priorité politique étant d'instaurer une politique méthodique fondée sur la répression d'une jeunesse considérée comme menaçante. Cette politique tend à délégitimer et à rendre invisibles les actions relevant d'un autre paradigme, et en particulier celui de la jeunesse comme ressource.

\section{Participation et expérimentation}

Si certains élus ont conscience de l'enjeu de la participation des jeunes et développent un travail de terrain pour les inciter à prendre part à la vie municipale, d'autres acceptent la participation des jeunes comme un devoir, sans forcement en anticiper les conséquences.

Une commune en a fait l'expérience: elle a mis en place un conseil local de la jeunesse, en confiant l'animation à une professionnelle et en oubliant ce que la jeunesse est capable de faire quand elle est accompagnée. J'ai démarré avec eux en avril 1999. J'ai fait un gros travail d'accompagnement sur l'analyse. Ils sont partis des rapports entre les jeunes et la police parce qu'ils habitent un quartier très tendu sur ces questions-là. Une partie d'entre eux sortent le soir, la nuit, et donc ils côtoient la Brigade anti-criminalité (BAC).

Le travail d'accompagnement suscite une véritable réflexion avec une enquête auprès de professionnels concernés par le sujet, qui va durer deux ans et qui va aboutir à la réalisation d'un film sur la garde-à-vue. La projection aura lieu sans la présence des élus municipaux qui ont décliné l'invitation. La publication d'un article dans le quotidien «Libération» va provoquer un choc au sein des responsables politiques. Les adultes se retrouvent face à une véritable participation de la jeunesse à laquelle ils ne s'attendaient pas. Le conflit qui suivra va avoir pour conséquence le départ de la coordinatrice du conseil de jeunes, mais aussi, va provoquer une véritable réflexion sur le rôle que doit jouer un conseil local de la jeunesse. Finalement, il y a eu une véritable rupture qui est due à la crainte de ces élus, à la non-connaissance de ces publics-là. Ils ignorent ce qui se passe, comment ça bouge, comment ça évolue. Pourtant, l'investissement est possible si on prend vraiment les jeunes au sérieux. Évidemment, il y a un conseil municipal qui est mis en place. C'est lui qui doit délibérer. Ça c'est normal, il ne faut pas modifier. Mais, s'il met en place un conseil de jeunes pour entendre son point de vue, et qu'il y a débat, c'est ça qui est important. Que le débat ait lieu.

On comprend bien que la création d'espaces de participations juvéniles demande une réflexion et une véritable volonté politique qui n'exclue pourtant pas la possibilité d'un conflit toujours possible lorsque l'échange existe vraiment. Celui-ci a souvent des effets positifs, entraînant la réflexion et donc l'évolution des positionnements de chacun vers la possibilité de l'exercice d'une citoyenneté juvénile.

Au-delà de l'engagement dans des conseils de jeunes, il existe d'autres formes de participations citoyennes par le biais d'associations, l'engagement dans des actions militantes ou des formations politiques. Moi j'étais membre du conseil local de jeunes à la commission «Droits». Mais j’étais aussi engagée politiquement. Du coup, il y a eu des élections municipales. Je me suis dit: «on va essayer de changer les choses de l'intérieur. J'y vais». Je ne pensais pas que ça allait être aussi compliqué que ça d'être conseillère municipale. Ce n'est pas facile de faire ça quand tu es black, que tu es une fille, même dans une ville de gauche!.

L'acte participatif de la jeunesse n'est jamais simple à mettre en place. Si la volonté politique est nécessaire, elle doit être englobée dans un processus de démocratisation plus large, touchant l'ensemble de la ville, notamment les populations étrangères extra-européennes qui n'ont pas accès au vote local. Pourtant, l'offre de participation citoyenne peut être instrumentalisée ce dont les jeunes sont conscients: je suis persuadée qu'on m'a 
prise sur cette liste parce que je suis jeune, black, issue d'un quartier... on ne m'a prise que pour ça. Après, moi, j'ai eu envie de me battre.

De leur côté, certains élus ont conscience de la difficulté de mettre en place une véritable relation, basée sur la confiance, avec les jeunes. La proximité ne veut pas dire que les jeunes ont toujours raison, il faut expliquer le bien fondé des choses. Ils expriment aussi la nécessité d'organiser l'arrivée et l'installation des jeunes au sein d'un conseil local. L'inscription dans une forme de participation citoyenne n'est pas simple et demande une certaine réflexion, pourtant, l'enjeu est important. Offrir un espace où puissent s'exprimer les savoirs et les savoir-faire de la jeunesse permet de construire une participation offrant la possibilité aux jeunes comme aux adultes de se rencontrer et de se connaître. De tâtonnements en expérimentations, les jeunes, en interaction avec des adultes responsables, peuvent envisager la construction d'une place citoyenne.

\section{Conclusion: la participation des jeunes, un remède à l'isolement politique}

Depuis les années 1980 marquées par les "rodéos des cités", puis les années 1990 et les "émeutes urbaines", les banlieues françaises subissent ce que l'on nomme désormais des “violences urbaines". Les événements de l'automne 2005 ont rassemblé les plus importantes agitations sociales et politiques depuis Mai 68 (Mucchielli, Goaziou, 2006). Loin des explications simplistes avancées par certaines formations politiques ou certains médias, accusant une immigration incontrôlée qui entretiendrait l'islamisation des banlieues, la démission des parents et une absence de civisme à la jeunesse, les causes sont à chercher dans la discrimination et la précarité que subissent les jeunes et leurs familles vivant dans les quartiers, mais aussi dans la remise en cause des droits de ces jeunes Français d'origine étrangère.

L'injustice du quotidien pousse les habitants des quartiers populaires vers une souffrance qui est à l'origine de la naissance de sentiments d'amertume et de révolte (Marliere, 2006). Cette position d'exclu plonge dans le silence - et parfois dans la violence - toute une population, empêchant toute possibilité d'échanges et de négociations. L'absence même d'un accès au conflit montre tout l'immobilisme dans lequel sont maintenus les habitants des quartiers. Certains jeunes, conscients de la nécessité d'acquérir une certaine visibilité, passent à l'acte en brûlant des voitures et en s'attaquant à des symboles de l'Etat. Selon H. Lagrange, ils trouvent là "un moyen de communication par défaut" (Lagrange et Oberti, 2006), face à une population qui reste silencieuse. En incendiant des écoles, ils trouvent aussi le moyen de montrer toute la frustration et l'expression des inégalités qui sont portées par ces lieux. En effet, ce chercheur nous rappelle que l'école publique et laïque française ne tient pas ses promesses d'intégration. Cette "panne institutionnelle" freine l'accès des jeunes au fonctionnement de la société, d'autant plus que l'Etat français pense la cohésion sociale et la citoyenneté au niveau national. Cette logique d'intégration de citoyens tous égaux ne peut fonctionner car chacun est traité, en réalité, de façon inégalitaire du point de vue spatial, social et ethnique. Les auteurs du présent article l'ont aussi constaté lors du travail de recherche sur "l'alternative jeunesse".

Pour conclure, revenons brièvement sur les causes des émeutes et les facteurs communs aux différents événements que les banlieues françaises ont connus depuis 1980. H. Lagrange en propose six qui correspondent à ce que nous avons pu entendre des jeunes participant à notre recherche-action. Tout d'abord, les émeutes se sont développées dans des quartiers d'habitat social classés en zones urbaines sensibles (ZUS) et présentant un contraste important avec le reste de la ville. Parmi ces ZUS, celles comprenant une forte proportion de jeunes âgés de moins de 20 ans ont été les plus impliquées dans les événements. Il constate que les villes où sont installées des familles originaires d'Afrique noire ont été très représentées dans les événements. Il explique cette constatation par les difficultés que rencontre ces familles, dernièrement arrivées, à migrer et à se socialiser, les enfants se retrouvant dans de mauvais parcours scolaires. À cela se rajoute, pour l'ensemble des villes concernées, le taux de chômage des jeunes qui reste une cause récurrente. En regardant de plus près les quartiers concernés par les émeutes, il constate qu'ils ont tous bénéficié de la politique de la ville en raison de leurs caractéristiques déficitaires (Lagrange et Oberti, 2006). Enfin, l'idée que ces quartiers sont au centre d'une politique dite de démolition/ 
/reconstruction, pilotée par l'Agence nationale de rénovation urbaine doit être prise en considération.

On retrouve bien dans les causes avancées ici la discrimination, la pauvreté, l'absence de perspectives dans la société française et la perte de confiance totale envers la République exposées dans le rapport des Renseignements Généraux commandé par le gouvernement à la suite des événements.

La concentration d'une population jeune dans des quartiers dégradés participe à l'apparition de sentiments d'insatisfaction, de frustration et d'inégalité. Les événements de novembre semblent donc bien portés par un ensemble de faits devenus inacceptables pour la jeunesse. La surdité politique oblige cette jeunesse à s'exprimer de façon radicale et violente alors qu'elle recherche un conflit constructif qu'on lui refuse.

Lors de notre travail avec le groupe de "l'alternative jeunesse", nous avons pu entendre la parole des jeunes que nous avons rendue publique grâce à un livre. D'autres chercheurs développent ce genre de démarche pour tenter d'exprimer les «maux» de la banlieue et de sa jeunesse. Pourtant, assourdis par le tintamarre sécuritaire, les responsables politiques semblent avoir des difficultés à comprendre le malaise profond qui court dans les banlieues. Une politique de contention s'appuyant sur le déploiement de forces de l'ordre semble être pratiquement toujours la seule solution proposée par la classe politique. Loin d'un processus de repli communautaire, la banlieue subit la montée d'un individualisme sociétal qui

\section{Bibliographie}

BACHMANN, Christian, LE GUENNEC, Nicole, 1996, Violences urbaines: ascension et chute des classes moyennes dans la politique de la ville, Paris, Albin Michel.

BACHMANN Christian, LE GUENNEC Nicole, 1997, Autopsie d'une émeute, Paris, Albin Michel.

BEAUD Stéphane., PIALOUX Michel, 2003, Violences urbaines, violences sociales: genèse des nouvelles classes dangereuses, Paris, Fayard.

BORDES, Véronique, VULBEAU, Alain., 2004 L'alternative jeunesse, Paris, L'Atelier/Profession Banlieue, collection Les savoirs de la ville. semble se généraliser. Dans ces zones qui deviennent des lieux de relégation pour une jeunesse populaire, l'accompagnement social reste primordial. Pourtant, les politiques actuelles tendent vers une désertification de la présence institutionnelle, enlevant aux associations leurs moyens d'action et laissant à la police le rôle de dernier contact avec les habitants.

Les émeutes permettent de percevoir le manque de reconnaissance et, selon l'expression de Hugues Lagrange, "l'isolement politique" d'une partie de la jeunesse qui ne peut plus participer au jeu social. Le processus des conseils de jeunes montre que, au contraire, lorsqu'une volonté politique existe, la participation des jeunes est possible: cette option favorise alors un désenclavement de la parole des jeunes et une entrée dans la citoyenneté.

Notre travail de recherche-action, homologue à cette démarche, espère avoir convaincu de la richesse d'une méthodologie qui permet, à son niveau, de porter la parole des jeunes vers une relative audience sociale. C'est alors aux décideurs de savoir s'ils veulent entendre la parole des jeunes et composer avec leur envie de participer et leur compétence pour construire en commun un avenir suffisamment satisfaisant pour tous. Comme l'a très bien souligné Michel Kokoreff (2003), l'enjeu est dans une repolitisation de l'expérience tenant compte de l'émergence de ces nouveaux acteurs que sont les jeunes des quartiers.

KOKOREFF, Michel, 2003, La force des quartiers: de la délinquance à l'engagement politique, Paris, Payot.

LAGRANGE, Hugues, OBERTI, Marc. (dir), 2006, Émeutes urbaines et protestation: une singularité française, Paris, Sciences Po.

MARLIERE, Eric., 2006, "Les habitants de quartiers: adversaires ou solidaires des émeutiers?" dans MUCCHIELLI, Laurent, LE GOAZIOU Véronique (dir), Quand les banlieues brûlent : retour sur les émeutes de novembre 2005, Paris, La Découverte.

MUCCHIELLI, Laurent, LE GOAZIOU Véronique (dir.), 2006, Quand les banlieues brûlent : retour sur les émeutes de novembre 2005, Paris, La Découverte.

VIEILLARD-BARON, Hervé, 2001, Les banlieue: des singularités françaises aux réalités mondiales, Paris, Hachette. 